\title{
Agegraphic Chaplygin gas model of dark energy
}

\author{
A. Sheykhi * \\ Department of Physics, Shahid Bahonar University, P.O. Box 76175, Kerman, Iran \\ Research Institute for Astronomy and Astrophysics of Maragha (RIAAM), Maragha, Iran
}

\begin{abstract}
We establish a connection between the agegraphic models of dark energy and Chaplygin gas energy density in non-flat universe. We reconstruct the potential of the agegraphic scalar field as well as the dynamics of the scalar field according to the evolution of the agegraphic dark energy. We also extend our study to the interacting agegraphic generalized Chaplygin gas dark energy model.
\end{abstract}

\section{INTRODUCTION}

Over the course of the past decade, evidence for the most striking result in modern cosmology has been steadily growing, namely the existence of an exotic dark energy component which has negative pressure and pushes the universe to accelerated expansion [1]. Of course, a natural explanation to the accelerated expansion is due to a positive tiny cosmological constant. Though, it suffers the so-called fine-tuning and cosmic coincidence problems. A great variety of scenarios have been proposed to explain this acceleration while most of them cannot explain all the features of universe or they have so many parameters that makes them difficult to fit. For a recent review on dark energy proposals see [2]. Many theoretical attempts toward understanding the dark energy problem are focused to shed light on it in the framework of a fundamental theory such as string theory or quantum gravity. Although a complete theory of quantum gravity has not established yet today, we still can make some attempts to investigate the nature of dark energy according to some principles of quantum gravity. The holographic dark energy model and the agegraphic dark energy model are just such examples, which are originated from some considerations of the features of the quantum theory of gravity. That is to say, the holographic and agegraphic dark energy models possess some significant features of quantum gravity. The former, that arose a lot of enthusiasm recently [3 9 ], is motivated from the holographic hypothesis [10] and has been tested and constrained by various astronomical observations [11]. However there are some difficulties in holographic dark energy model. Choosing the event horizon of the universe as the length scale, the holographic dark energy gives the observation value of dark energy in the universe and can drive the universe to an accelerated expansion phase. But an obvious drawback concerning causality appears in this proposal. Event horizon is a global concept of spacetime; existence of event horizon of the universe depends on future evolution of the universe; and event horizon exists only for universe with forever accelerated expansion. In addition, more recently, it has been argued that this proposal might be in contradiction to the age of some old high redshift objects, unless a lower Hubble parameter is considered [12]. The later (agegraphic dark energy) is based on the uncertainty relation of quantum mechanics together with the gravitational effect in general relativity. The agegraphic dark energy model assumes that the observed dark energy comes from the spacetime and matter field fluctuations in the universe [13 15]. Since in agegraphic dark energy model the age of the universe is chosen as the length measure, instead of the horizon distance, the causality problem in the holographic dark energy is avoided. The agegraphic models of dark energy have been examined and constrained by various astronomical observations [16 18].

Among the various candidates to explain the accelerated expansion, the Chaplygin gas dark energy model has emerged as a possible unification of dark matter and dark energy, since its cosmological evolution is similar to an initial dust like matter and a cosmological constant for late times. Inspired by the fact that the Chaplygin gas possesses a negative pressure, the authors of [19] have undertaken the simple task of studying a FRW cosmology of a universe filled with this type of fluid. The equation of state of the Chaplygin gas dark energy obeys 20]

$$
p_{D}=\frac{-A}{\rho_{D}},
$$

where $\rho_{D}>0$ and $p_{D}$ are, respectively, the energy density and pressure of Chaplygin gas dark energy, and $A$ is a positive constant. This equation of state has raised a certain interest [21] because of its many interesting and, in some sense, intriguingly unique features. Some possible motivations for this model from the field theory points of view are investigated in 22]. The Chaplygin gas emerges as an effective fluid associated with D-branes [23] and can also be obtained from the Born-Infeld action 24]. The connection between the holographic models of dark energy and the Chaplygin gas energy density has been established in [25, 26].

*sheykhi@mail.uk.ac.ir 
Our aim in this Letter is to establish a correspondence between the agegraphic dark energy scenarios and the Chaplygin gas model. We suggest the agegraphic description of the Chaplygin gas dark energy in FRW universe and reconstruct the potential and the dynamics of the scalar field which describe the Chaplygin cosmology. In the next section we study the original agegraphic Chaplygin gas model. In section III, we establish the correspondence between the new model of agegraphic dark energy and the Chaplygin gas dark energy. In section IV we extend our study to the interacting agegraphic generalized Chaplygin gas dark energy model. The last section is devoted to conclusions.

\section{THE ORIGINAL ADE AS A CHAPLYGIN GAS}

We assume the agegraphic Chaplygin gas dark energy is accommodated in the Friedmann-Robertson-Walker (FRW) universe which is described by the line element

$$
d s^{2}=d t^{2}-a^{2}(t)\left(\frac{d r^{2}}{1-k r^{2}}+r^{2} d \Omega^{2}\right),
$$

where $a(t)$ is the scale factor, and $k$ is the curvature parameter with $k=-1,0,1$ corresponding to open, flat, and closed universes, respectively. A closed universe with a small positive curvature $\left(\Omega_{k} \simeq 0.01\right)$ is compatible with observations [27]. The corresponding Friedmann equation takes the form

$$
H^{2}+\frac{k}{a^{2}}=\frac{1}{3 m_{p}^{2}}\left(\rho_{m}+\rho_{D}\right) .
$$

We introduce, as usual, the fractional energy densities such as

$$
\Omega_{m}=\frac{\rho_{m}}{3 m_{p}^{2} H^{2}}, \quad \Omega_{D}=\frac{\rho_{D}}{3 m_{p}^{2} H^{2}}, \quad \Omega_{k}=\frac{k}{H^{2} a^{2}},
$$

thus, the Friedmann equation can be written

$$
\Omega_{m}+\Omega_{D}=1+\Omega_{k}
$$

Inserting the equation of state (1) into the relativistic energy conservation equation, leads to a density evolving as

$$
\rho_{D}=\sqrt{A+\frac{B}{a^{6}}}
$$

where $B$ is an integration constant. We adopt the viewpoint that the scalar field models of dark energy are effective theories of an underlying theory of dark energy. The energy density and pressure of the scalar field can be written as

$$
\begin{aligned}
& \rho_{\phi}=\frac{1}{2} \dot{\phi}^{2}+V(\phi)=\sqrt{A+\frac{B}{a^{6}}}, \\
& p_{\phi}=\frac{1}{2} \dot{\phi}^{2}-V(\phi)=\frac{-A}{\sqrt{A+\frac{B}{a^{6}}}},
\end{aligned}
$$

Then, we can easily obtain the scalar potential and the kinetic energy term as

$$
\begin{aligned}
& V(\phi)=\frac{2 A a^{6}+B}{2 a^{6} \sqrt{A+\frac{B}{a^{6}}}}, \\
& \dot{\phi}^{2}=\frac{B}{a^{6} \sqrt{A+\frac{B}{a^{6}}}} .
\end{aligned}
$$

Now we are focussing on the reconstruction of the original agegraphic Chaplygin gas model of dark energy. Let us first review the origin of the agegraphic dark energy. Following the line of quantum fluctuations of spacetime, Karolyhazy et al. [28] argued that the distance $t$ in Minkowski spacetime cannot be known to a better accuracy than $\delta t=\beta t_{p}^{2 / 3} t^{1 / 3}$ where $\beta$ is a dimensionless constant of order unity. Based on Karolyhazy relation, Maziashvili discussed that the energy density of metric fluctuations of the Minkowski spacetime is given by [29]

$$
\rho_{D} \sim \frac{1}{t_{p}^{2} t^{2}} \sim \frac{m_{p}^{2}}{t^{2}}
$$


where $t_{p}$ is the reduced Planck time. We use the units $c=\hbar=k_{b}=1$ throughout this Letter. Therefore one has $l_{p}=t_{p}=1 / m_{p}$ with $l_{p}$ and $m_{p}$ are the reduced Planck length and mass, respectively. The original agegraphic dark energy density has the form (11) where $t$ is chosen to be the age of the universe

$$
T=\int_{0}^{a} \frac{d a}{H a},
$$

Thus, the energy density of the original agegraphic dark energy is given by [13]

$$
\rho_{D}=\frac{3 n^{2} m_{p}^{2}}{T^{2}}
$$

where the numerical factor $3 n^{2}$ is introduced to parameterize some uncertainties, such as the species of quantum fields in the universe, the effect of curved space-time (since the energy density is derived for Minkowski space-time), and so on. The dark energy density (13) has the same form as the holographic dark energy, but the length measure is chosen to be the age of the universe instead of the horizon radius of the universe. Thus the causality problem in the holographic dark energy is avoided. Using Eqs. (4) and (13), we have

$$
\Omega_{D}=\frac{n^{2}}{H^{2} T^{2}}
$$

We assume the agegraphic dark energy and dark matter evolve according to their conservation laws

$$
\begin{aligned}
& \dot{\rho}_{D}+3 H \rho_{D}\left(1+w_{D}\right)=0, \\
& \dot{\rho}_{m}+3 H \rho_{m}=0,
\end{aligned}
$$

where $w_{D}$ is the equation of state parameter of agegraphic dark energy. Taking the derivative with respect to the cosmic time of Eq. (13) and using Eq. (14) we get

$$
\dot{\rho}_{D}=-2 H \frac{\sqrt{\Omega_{D}}}{n} \rho_{D}
$$

Inserting this relation into Eq. (15), we obtain the equation of state parameter of the original agegraphic dark energy

$$
w_{D}=-1+\frac{2}{3 n} \sqrt{\Omega_{D}} .
$$

Differentiating Eq. (14) and using relation $\dot{\Omega}_{D}=\Omega_{D}^{\prime} H$, we reach

$$
\Omega_{D}^{\prime}=\Omega_{D}\left(-2 \frac{\dot{H}}{H^{2}}-\frac{2}{n} \sqrt{\Omega_{D}}\right),
$$

where the dot is the derivative with respect to the cosmic time and the prime denotes the derivative with respect to $x=\ln a$. Taking the derivative of both side of the Friedman equation (3) with respect to the cosmic time, and using Eqs. (5), (13), (14) and (16), it is easy to find

$$
\frac{\dot{H}}{H^{2}}=-\frac{3}{2}\left(1-\Omega_{D}\right)-\frac{\Omega_{D}^{3 / 2}}{n}-\frac{\Omega_{k}}{2} .
$$

Substituting this relation into Eq. (19), we obtain the equation of motion of agegraphic dark energy

$$
\Omega_{D}^{\prime}=\Omega_{D}\left[\left(1-\Omega_{D}\right)\left(3-\frac{2}{n} \sqrt{\Omega_{D}}\right)+\Omega_{k}\right] .
$$

Next, we establish the connection between the original agegraphic dark energy and Chaplygin gas energy density. Combining Eqs. (6) and (13), we obtain

$$
B=a^{6}\left(9 n^{4} m_{p}^{4} T^{-4}-A\right) .
$$

Using Eqs. (11), (6) and (18) one can write

$$
w_{D}=\frac{-A}{\rho_{D}^{2}}=\frac{-A}{A+\frac{B}{a^{6}}}=-1+\frac{2}{3 n} \sqrt{\Omega_{D}} .
$$


Substituting $B$ in the above equation, we obtain following relation for $A$ :

$$
A=9 n^{4} m_{p}^{4} T^{-4}\left(1-\frac{2}{3 n} \sqrt{\Omega_{D}}\right) .
$$

Therefore the constant $B$ is given by

$$
B=6 a^{6} n^{4} m_{p}^{4} T^{-4} \frac{\sqrt{\Omega_{D}}}{n} .
$$

Finally, we rewrite the scalar potential and kinetic energy term as

$$
\begin{aligned}
V(\phi) & =n^{2} m_{p}^{2} T^{-2}\left(3-\frac{\sqrt{\Omega_{D}}}{n}\right)=m_{p}^{2} H^{2} \Omega_{D}\left(3-\frac{\sqrt{\Omega_{D}}}{n}\right), \\
\dot{\phi} & =n m_{p} T^{-1} \sqrt{\frac{2}{n} \Omega_{D}^{1 / 2}}=m_{p} H \sqrt{\frac{2}{n} \Omega_{D}^{3 / 2}} .
\end{aligned}
$$

Using relation $\dot{\phi}=H \phi^{\prime}$, we get

$$
\phi^{\prime}=m_{p} \sqrt{\frac{2}{n} \Omega_{D}^{3 / 2}} .
$$

Consequently, we can easily obtain the evolution behavior of the scalar field

$$
\phi(a)-\phi\left(a_{0}\right)=\int_{a_{0}}^{a} \frac{m_{p}}{a} \sqrt{\frac{2}{n} \Omega_{D}^{3 / 2}} d a,
$$

where $a_{0}$ is the present value of the scale factor, and $\Omega_{D}$ can be obtained through Eq. (21).

\section{THE NEW ADE AS A CHAPLYGIN GAS}

Soon after the original agegraphic dark energy model was introduced by Cai [13], a new model of agegraphic dark energy was proposed in [14], while the time scale is chosen to be the conformal time $\eta$ instead of the age of the universe. This new agegraphic dark energy contains some new features different from the original agegraphic dark energy and overcome some unsatisfactory points. For instance, the original agegraphic dark energy suffers from the difficulty to describe the matter-dominated epoch while the new agegraphic dark energy resolved this issue [14]. The energy density of the new agegraphic dark energy can be written

$$
\rho_{D}=\frac{3 n^{2} m_{p}^{2}}{\eta^{2}}
$$

where the conformal time is given by

$$
\eta=\int \frac{d t}{a}=\int_{0}^{a} \frac{d a}{H a^{2}} .
$$

The fractional energy density of the new agegraphic dark energy is now given by

$$
\Omega_{D}=\frac{n^{2}}{H^{2} \eta^{2}} .
$$

Taking the derivative with respect to the cosmic time of Eq. (30) and using Eq. (32) we get

$$
\dot{\rho}_{D}=-2 H \frac{\sqrt{\Omega_{D}}}{n a} \rho_{D} .
$$

Inserting this relation into Eq. (15) we obtain the equation of state parameter of the new agegraphic dark energy

$$
w_{D}=-1+\frac{2}{3 n a} \sqrt{\Omega_{D}} .
$$


Then we obtain, following the approach of the previous section, the evolution behavior of the new agegraphic dark energy,

$$
\Omega_{D}^{\prime}=\Omega_{D}\left[\left(1-\Omega_{D}\right)\left(3-\frac{2}{n a} \sqrt{\Omega_{D}}\right)+\Omega_{k}\right] .
$$

Next, we construct the new agegraphic Chaplygin gas model, connecting the Chaplygin gas model with new agegraphic dark energy. Identifying Eq. (6) with (30) we have

$$
B=a^{6}\left(9 n^{4} m_{p}^{4} \eta^{-4}-A\right)
$$

Using Eqs. (1), (6) and (34), we reach

$$
w_{D}=\frac{-A}{A+\frac{B}{a^{6}}}=-1+\frac{2}{3 n a} \sqrt{\Omega_{D}} .
$$

Combining this equation with Eq. (36), we obtain

$$
\begin{aligned}
& A=9 n^{4} m_{p}^{4} \eta^{-4}\left(1-\frac{2}{3 n a} \sqrt{\Omega_{D}}\right), \\
& B=6 a^{6} n^{4} m_{p}^{4} \eta^{-4} \frac{\sqrt{\Omega_{D}}}{n a} .
\end{aligned}
$$

Finally, we reconstruct the scalar potential and kinetic energy term as

$$
\begin{aligned}
V(\phi) & =n^{2} m_{p}^{2} \eta^{-2}\left(3-\frac{\sqrt{\Omega_{D}}}{n a}\right)=m_{p}^{2} H^{2} \Omega_{D}\left(3-\frac{\sqrt{\Omega_{D}}}{n a}\right) \\
\dot{\phi} & =n m_{p} \eta^{-1} \sqrt{\frac{2}{n a} \Omega_{D}^{1 / 2}}=m_{p} H \sqrt{\frac{2}{n a} \Omega_{D}^{3 / 2}} .
\end{aligned}
$$

Eq. (41) can also be reexpressed as

$$
\phi^{\prime}=m_{p} \sqrt{\frac{2}{n a} \Omega_{D}^{3 / 2}} .
$$

Therefore, we can obtain the evolutionary form of the scalar field

$$
\phi(a)-\phi\left(a_{0}\right)=\int_{a_{0}}^{a} \frac{m_{p}}{a} \sqrt{\frac{2}{n a} \Omega_{D}^{3 / 2}} d a,
$$

where $\Omega_{D}$ can be derived from Eq. (35).

\section{INTERACTING NEW AGEGRAPHIC GENERALIZED CHAPLYGIN GAS}

In this section we extend our study to the generalized Chaplygin gas when there is an interaction between generalized Chaplygin gas energy density and dark matter. The total energy density is $\rho=\rho_{m}+\rho_{D}$, where $\rho_{m}$ and $\rho_{D}$ are the energy density of dark matter and dark energy, respectively. The total energy density satisfies a conservation law

$$
\dot{\rho}+3 H(\rho+p)=0 .
$$

However, since we consider the interaction between dark matter and dark energy, $\rho_{m}$ and $\rho_{D}$ do not conserve separately; they must rather enter the energy balances

$$
\begin{aligned}
& \dot{\rho}_{m}+3 H \rho_{m}=Q, \\
& \dot{\rho}_{D}+3 H \rho_{D}\left(1+w_{D}\right)=-Q,
\end{aligned}
$$

where $Q$ denotes the interaction term and can be taken as $Q=3 b^{2} H \rho$ with $b^{2}$ being a coupling constant. In the generalized Chaplygin gas approach [24], the equation of state (11) is generalized to

$$
p_{D}=\frac{-A}{\rho_{D}^{\alpha}} \text {. }
$$


The above equation of state leads to a density evolution as

$$
\rho_{D}=\left(A+B a^{-3 \beta}\right)^{1 / \beta},
$$

where $\beta=\alpha+1$. Thus we have

$$
w_{D}=\frac{p_{D}}{\rho_{D}}=\frac{-A}{A+B a^{-3 \beta}} .
$$

Inserting relation (33) into Eq. (46) and using Eqs. (40) and (5), we obtain the equation of state parameter of the interacting new agegraphic dark energy

$$
w_{D}=-1+\frac{2}{3 n a} \sqrt{\Omega_{D}}-\frac{b^{2}}{\Omega_{D}}\left(1+\Omega_{k}\right) .
$$

The evolution behavior of the new agegraphic dark energy is given by

$$
\Omega_{D}^{\prime}=\Omega_{D}\left[\left(1-\Omega_{D}\right)\left(3-\frac{2}{n a} \sqrt{\Omega_{D}}\right)-3 b^{2}\left(1+\Omega_{k}\right)+\Omega_{k}\right] .
$$

We now establish the correspondence between the new agegraphic dark energy and generalized Chaplygin gas energy density. Identifying Eq. (30) with Eq. (48) and using Eq. (32) we get

$$
\left(3 m_{p}^{2} H^{2} \Omega_{D}\right)^{\beta}=A+B a^{-3 \beta} .
$$

Combining Eqs. (49) and (50) we find

$$
A=-\left(A+B a^{-3 \beta}\right)\left[-1+\frac{2}{3 n a} \sqrt{\Omega_{D}}-\frac{b^{2}}{\Omega_{D}}\left(1+\Omega_{k}\right)\right] .
$$

Solving Eqs. (52) and (53) we obtain

$$
\begin{aligned}
& A=\left(3 m_{p}^{2} H^{2} \Omega_{D}\right)^{\beta}\left[1-\frac{2}{3 n a} \sqrt{\Omega_{D}}+\frac{b^{2}}{\Omega_{D}}\left(1+\Omega_{k}\right)\right], \\
& B=\left(3 m_{p}^{2} H^{2} \Omega_{D} a^{3}\right)^{\beta}\left[\frac{2}{3 n a} \sqrt{\Omega_{D}}-\frac{b^{2}}{\Omega_{D}}\left(1+\Omega_{k}\right)\right] .
\end{aligned}
$$

Next we regard the scalar field model as an effective description of an underlying theory of dark energy with energy density and pressure

$$
\begin{aligned}
& \rho_{\phi}=\frac{1}{2} \dot{\phi}^{2}+V(\phi)=\left(A+B a^{-3 \beta}\right)^{1 / \beta}, \\
& p_{\phi}=\frac{1}{2} \dot{\phi}^{2}-V(\phi)=-A\left(A+B a^{-3 \beta}\right)^{-\alpha / \beta},
\end{aligned}
$$

where we have identified $\rho_{\phi}$ with $\rho_{D}$. Substituting $A$ and $B$ into Eqs. (56) and (57) one can easily find the scalar potential and the kinetic energy term as

$$
\begin{aligned}
V(\phi) & =m_{p}^{2} H^{2} \Omega_{D}\left(3-\frac{\sqrt{\Omega_{D}}}{n a}+\frac{3 b^{2}}{2} \frac{\left(1+\Omega_{k}\right)}{\Omega_{D}}\right), \\
\dot{\phi} & =m_{p} H\left(\frac{2}{n a} \Omega_{D}^{3 / 2}-3 b^{2}\left(1+\Omega_{k}\right)\right)^{1 / 2} .
\end{aligned}
$$

Using Eq. (59), Eq. (58) can be reexpressed as

$$
V(\phi)=3 m_{p}^{2} H^{2} \Omega_{D}\left(1-\frac{\dot{\phi}^{2}}{6 m_{p}^{2} H^{2} \Omega_{D}}\right) .
$$

We can also rewrite Eq. (59) as

$$
\phi^{\prime}=m_{p}\left(\frac{2}{n a} \Omega_{D}^{3 / 2}-3 b^{2}\left(1+\Omega_{k}\right)\right)^{1 / 2} .
$$


Consequently, we can easily obtain the evolutionary form of the field by integrating the above equation. The result is

$$
\phi(a)-\phi\left(a_{0}\right)=\int_{a_{0}}^{a} \frac{m_{p}}{a} \sqrt{\frac{2}{n a} \Omega_{D}^{3 / 2}-3 b^{2}\left(1+\Omega_{k}\right)} d a,
$$

where $\Omega_{D}$ is given by Eq. (51). In this way we connect the interacting new agegraphic dark energy with a generalized Chaplygin gas energy density and reconstruct the potential of the agegraphic Chaplygin gas.

\section{CONCLUSIONS}

In this Letter, we have established a correspondence between the agegraphic dark energy scenarios and the Chaplygin gas model of dark energy in non-flat FRW cosmology. A so-called agegraphic dark energy model has been proposed recently by Cai [13], based on the uncertainty relation of quantum mechanics together with the gravitational effect in general relativity. Since the original agegraphic dark energy model suffers from the difficulty to describe the matterdominated epoch, a new model of agegraphic dark energy was proposed in [14] while the time scale is chosen to be the conformal time $\eta$ instead of the age of the universe. We have adopted the viewpoint that the scalar field models of dark energy are effective theories of an underlying theory of dark energy. If we regard the scalar field model as an effective description of such a theory, we should be capable of using the scalar field model to mimic the evolving behavior of the agegraphic dark energy and reconstructing this scalar field model according to the evolutionary behavior of agegraphic dark energy. We have reconstructed the potential of the agegraphic scalar field as well as the dynamics of the scalar field which describe the Chaplygin cosmology. Finally, we have extended our study to the agegraphic generalized Chaplygin gas dark energy model when there is an interaction between generalized Chaplygin gas energy density and dark matter.

\section{Acknowledgments}

This work has been supported by Research Institute for Astronomy and Astrophysics of Maragha.

[1] A.G. Riess, et al., Astron. J. 116 (1998) 1009;

S. Perlmutter, et al., Astrophys. J. 517 (1999) 565;

S. Perlmutter, et al., Astrophys. J. 598 (2003) 102;

P. de Bernardis, et al., Nature 404 (2000) 955.

[2] T. Padmanabhan, Phys. Rep. 380 (2003) 235;

P. J. E. Peebles, B. Ratra, Rev. Mod. Phys. 75 (2003) 559;

E. J. Copeland, M. Sami and S. Tsujikawa, hep-th/060305.

[3] A. Cohen, D. Kaplan, A. Nelson, Phys. Rev. Lett. 82 (1999) 4971.

[4] M. Li, Phys. Lett. B 603 (2004) 1.

[5] Q. G. Huang, M. Li, JCAP 0408 (2004) 013.

[6] S. D. H. Hsu, Phys. Lett. B 594 (2004) 13.

[7] E. Elizalde, S. Nojiri, S.D. Odintsov, P. Wang, Phys. Rev. D 71 (2005) 103504;

B. Guberina, R. Horvat, H. Stefancic, JCAP 0505 (2005) 001;

B. Guberina, R. Horvat, H. Nikolic, Phys. Lett. B 636 (2006) 80;

H. Li, Z. K. Guo, Y. Z. Zhang, Int. J. Mod. Phys. D 15 (2006) 869;

Q. G. Huang, Y. Gong, JCAP 0408 (2004) 006;

J. P. B. Almeida, J. G. Pereira, Phys. Lett. B 636 (2006) 75;

Y. Gong, Phys. Rev. D 70 (2004) 064029;

B. Wang, E. Abdalla, R. K. Su, Phys. Lett. B 611 (2005) 21;

J.Y . Shen, B. Wang, E. Abdalla, R. K. Su, Phys. Lett. B 609 (2005) 200.

[8] M. R. Setare, Phys. Lett. B 642 (2006) 1;

M. R. Setare, S. Shafei, JCAP 09 (2006) 011;

M. R. Setare, E. C. Vagenas, Phys. Lett. B 666 (2008) 111;

H. M. Sadjadi, arXiv:0902.2462

M. R. Setare, E. N. Saridakis, Phys. Lett. B 671 (2009) 331;

M. Jamil, E. N. Saridakis, M. R. Setare, arXiv: 0906.2847. 
[9] M. R. Setare, Eur. Phys. J. C 50 (2007) 991;

M. R. Setare, JCAP 0701 (2007) 023;

M. R. Setare, Phys. Lett. B 642 (2006) 421.

[10] G. t Hooft, gr-qc/9310026. L. Susskind, J. Math. Phys. 36 (1995) 6377.

[11] X. Zhang, F. Q. Wu, Phys. Rev. D 72 (2005) 043524;

X. Zhang, F. Q. Wu, Phys. Rev. D 76 (2007) 023502;

Q. G. Huang, Y.G. Gong, JCAP 0408 (2004) 006;

K. Enqvist, S. Hannestad, M. S. Sloth, JCAP 0502 (2005) 004;

J.Y. Shen, B. Wang, E. Abdalla, R.K. Su, Phys. Lett. B 609 (2005) 200;

Q. Wu, et al., arXiv:0705.1006.

[12] H. Wei and S. N. Zhang, arXiv:0707.2129

[13] R. G. Cai, Phys. Lett. B 657 (2007) 228.

[14] H. Wei and R. G. Cai, Phys. Lett. B 660 (2008) 113.

[15] H. Wei and R. G. Cai, Eur. Phys. J. C 59 (2009) 99.

[16] H. Wei and R. G. Cai, Phys. Lett. B 663 (2008) 1;

J. Cui, et al., arXiv:0902.0716.

Y. W. Kim, et al., Mod. Phys. Lett. A 23 (2008) 3049;

Y. Zhang, et al. arXiv:0708.1214;

J .P Wu, D. Z. Ma, Y. Ling, Phys. Lett. B 663, (2008) 152;

K. Y. Kim, H. W. Lee, Y. S. Myung, Phys.Lett. B 660 (2008) 118;

X. Wu, et al., arXiv:0708.0349.

J. Zhang, X. Zhang, H. Liu, Eur. Phys. J. C 54 (2008) 303;

I. P. Neupane, Phys. Lett. B 673 (2009) 111.

[17] A. Sheykhi, arXiv:0907.5144,

A. Sheykhi, 0908.0606.

[18] M. R. Setare, arXiv:0907.4910.

M. R. Setare, arXiv:0908.0196

[19] V. Gorini, A. Kamenshchik, U. Moschella, V. Pasquier, gr-qc/0403062

[20] A.Yu. Kamenshchik, U. Moschella, V. Pasquier, Phys. Lett. B 511 (2001) 265.

[21] D. Bazeia, R. Jackiw, Ann. Phys. 270 (1998) 246;

D. Bazeia, Phys. Rev. D 59 (1999) 085007;

R. Jackiw, A.P. Polychronakos, Commun. Math. Phys. 207 (1999) 107;

N. Ogawa, Phys. Rev. D 62 (2000) 085023.

[22] N. Bilic, G.B. Tupper, R.D. Viollier, Phys. Lett. B 535 (2002) 17;

N. Bilic, G.B. Tupper, R.D. Viollier, astro-ph/0207423.

[23] M. Bordemann, J. Hoppe, Phys. Lett. B 317 (1993) 315;

J.C. Fabris, S.V.B. Gonsalves, P.E. de Souza, Gen. Relativ. Gravit. 34 (2002) 53.

[24] M.C. Bento, O. Bertolami, A.A. Sen, Phys. Lett. B 575 (2003) 172.

[25] M. R. Setare, Phys. Lett. B 648 (2007) 329.

[26] M. R. Setare, Eur. Phys. J. C 52 (2007) 689;

M. R. Setare, Phys. Lett. B 654 (2007) 1;

M. R. Setare, Int. J. Mod. Phys. D 18 (2009) 419.

[27] D. N. Spergel, Astrophys. J. Suppl. 148 (2003) 175;

C. L. Bennett, et al., Astrophys. J. Suppl. 148 (2003) 1;

U. Seljak, A. Slosar, P. McDonald, JCAP 0610 (2006) 014;

D. N. Spergel, et al., Astrophys. J. Suppl. 170 (2007) 377.

[28] F. Karolyhazy, Nuovo.Cim. A 42 (1966) 390;

F. Karolyhazy, A. Frenkel and B. Lukacs, in Physics as natural Philosophy edited by A. Shimony and H. Feschbach, MIT Press, Cambridge, MA, (1982);

F. Karolyhazy, A. Frenkel and B. Lukacs, in Quantum Concepts in Space and Time edited by R. Penrose and C.J. Isham, Clarendon Press, Oxford, (1986).

[29] M. Maziashvili Int. J. Mod. Phys. D 16 (2007) 1531;

M. Maziashvili, Phys. Lett. B 652 (2007) 165. 